\title{
Gemeinnützigkeit, soziale Verantwortung und wirtschaftlicher Erfolg
}

\section{Zur Position des Managements in sozialen Organisationen}

Immer wieder gelangen Fälle aus gemeinnützigen Organisationen an die Öffentlichkeit, in denen die Geschäftsgebaren des Managements mit dem altruistischen Profil, das mit diesen Organisationen verbunden wird, in Konflikt zu stehen scheinen. Diskutiert werden in diesem Zusammenhang einerseits juristische Voraussetzungen der Gemeinnützigkeit und andererseits die soziale und ökonomische Nachhaltigkeit vor dem Hintergrund des wahrgenommenen "Mission Drift".

$\mathrm{D}$ er Leiter einer gemeinnützigen $\mathrm{GmbH}$ in Berlin, die sich in der Obdachlosen-Hilfe engagierte, sorgte 2010 für Empörung, weil er einen Maserati als Dienstwagen nutzte. Auseinandersetzungen mit seinem Gehalt und weiteren Vergünstigungen, etwa einer Dienstwohnung, folgten. Der Leiter verwehrte sich gegen entsprechende Vorwürfe mit der Begründung: „Wir sind kein kleiner Verein mehr (...). Sozialwirtschaft ist heute auch einfach Wirtschaft. Da ist auch die Außenwirkung einer Hilfsorganisation wichtig" (Jüttner 2010). Sein Gehalt und sonstige Vergüns-

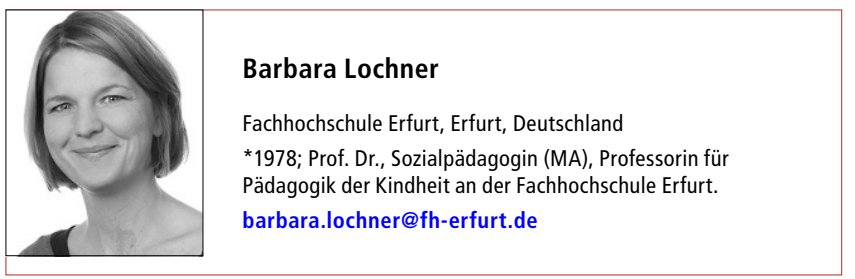

Jörg Altmann
$\begin{aligned} & \text { Fachhochschule Erfurt, Erfurt, Deutschland } \\ & \text { *1971; Prof. Dr., Jurist, Professor für Recht der Sozialen Arbeit an } \\ & \text { der Fachhochschule Erfurt. } \\ & \text { joerg.altmann@fh-erfurt.de }\end{aligned}$

Zusammenfassung Der Beitrag geht der Frage nach, inwiefern sich gemeinnützige Organisationen als wirtschaftliche Akteure positionieren (können) und welche Konsequenzen sich daraus für das Management ergeben.

Schlüsselwörter Wohlfahrtsverbände, Gemeinnützigkeit, Corporate Social Responsibility, Soziale Verantwortung tigungen wären vor dem Hintergrund seines äußerst erfolgreichen Managements gerechtfertigt (ebd.). Dieser Argumentation wurde von Politik und Öffentlichkeit nicht gefolgt und auch das Finanzamt erkannt 2011 der gGmbH die Gemeinnützigkeit ab (Schönball 2011). Das Land Berlin kündigte laut eines Zeitungsartikels Aufträge im Umfang von fünf Millionen Euro und Ende 2011 meldete die Organisation Insolvenz an (Reinhardt 2014).

Knapp zehn Jahre später sorgt in Thüringen die Argumentationsweise der Geschäftsführung der AWO AJS gGmbH für ähnliche Aufregung. Auch hier wird diskutiert, ob Gehalt, Dienstwagengröße und Vergünstigungen der Geschäftsführung in Einklang zu bringen sind mit der gesellschaftlichen und sozialen Position einer gemeinnützigen Organisation der Wohlfahrtspflege. Der Bundesverband der AWO leitet Untersuchungen gegen den Thüringer Verband ein, weil er die Grundwerte der AWO gefährdet sieht. "Wir wollen die Marke Arbeiterwohlfahrt schützen und erhalten “, so Wolfgang Stadler vom AWO-Bundesverband in einem Interview $\mathrm{zu}$ der Auseinandersetzung mit dem Thüringer Landesverband (Reinhardt und Reißland 2020). Die Geschäftsführung sieht die Vergütung hingegen im Erfolg der Organisation begründet. Die AWO AJS gGmbH sei eine Organisation mit mehr als 5000 Mitarbeiter_innen in 200 Einrichtungen und einem Jahresumsatz von etwa 220 Mio. €. In einer Pressemitteilung wird erklärt: „Die Gehälter der Geschäftsführung wurden durch die Gesellschaftervertreter zum Teil lange vor der Einführung des Governance Kodex [des AWO Bundesverbandes, BL] und auf Grundlage klarer Kriterien verhandelt: Größe des Unternehmens, Mitarbeiterzahl, Zahl 
der Einrichtungen, Umsatzerlöse, Erfolg des Unternehmens, Erfahrung und Kompetenz der Geschäftsführung “ (AWO AJS gGmbH 2020).

Die Auseinandersetzung mit der Frage, ob in sozialen Organisationen die gleichen Maßstäbe für die Gehälter des Managements herangezogen werden können wie in Wirtschaftsbetrieben, beschränkt sich nicht auf die hier skizzierten Fälle. Dass „Verbände [...] sich immer häufiger als Sozialunternehmen [definieren] und [man] immer häufiger [...] auch die Statussymbole der gewerblichen Wirtschaft bei ihren Führungskräften [findet] “ (Vilain 2008, S. 154), begründet sich in Prozessen der Ökonomisierung, die bereits in den 1990er-Jahren begannen. Sie werden mit dem Argument der Professionalisierung sozialer Dienstleistungen verknüpft, worunter überwiegend ein an Effizienz und Effektivität ausgerichtetes Management verstanden wird. Die Manager_innen dieser Sozialunternehmen postulieren, dass sie sich am unternehmerischen Erfolg der Organisationen messen lassen wollen und fordern, dass sich ihre Gehälter und Privilegien entsprechend an wirtschaftlichen Gepflogenheiten orientieren sollten. Im Governance-Kodex des AWO-Bundesverbandes wird hingegen für eine Orientierung an den Gehältern im öffentlichen Dienst und eine angemessene Bestimmung im Rahmen des organisationsinternen Gehaltsgefüges plädiert - was im Kern die Honorierung eines durch Einsparung von Personalkosten erzielten Unternehmenserfolgs (vgl. Bastian 2018, S. 22f.) erschwert. In Thüringen legen die drei 2021 regierenden Landtagsfraktionen (Linke, SPD, Bündnis 90/Die Grünen) einen Antrag vor, mit dem ein Dialogprozess zwischen Politik und Thüringer Sozialwirtschaft angeregt werden soll, um ein „Leitbild Subsidiarität und Transparenz“ zu entwickeln. Begründet wird dieses Ansinnen damit, dass „[d]as Wesen dieser Organisationen, die mit sozialen, politischen und gesellschaftlichen Wertvorstellungen verbunden sind, [...] eine besondere Verantwortung ein[schließt]. Sie erfordert ein besonderes Maß an Offenheit und Transparenz gegenüber der Gesellschaft und den Geldgeberinnen und Geldgebern dieser Leistungen. [...] Ein starkes Gehaltsgefälle zwischen Führungskräften einerseits und den Mitarbeiterinnen und Mitarbeitern andererseits ist nicht hinnehmbar“ (Linke, SPD, Bündnis 90/Die Grünen 2021).

Wie stichhaltig ist die Einschätzung, dass an die Positionierung des Managements von sozialen Organisationen andere Maßstäbe angelegt werden sollten, als an jenes von Wirtschaftsunternehmen? Dieser Frage soll im Folgenden aus juristischer und sozioökonomischer Perspektive nachgegangen werden.
Ökonomischer Handlungsspielraum im Kontext des Gemeinnützigkeitsrecht

Inwieweit eine wirtschaftliche Betätigung und ökonomisches/unternehmerisches Handeln in sozialen Arbeitsfeldern mit der Gemeinnützigkeit vereinbar ist, beschäftigt die politische und fachliche Diskussion und auch die Gerichte und Finanzämter in regelmäßigen Abständen. Grundsätzlich ist die zuvor zitierte Aussage „Sozialwirtschaft ist heute auch einfach Wirtschaft" richtig, denn in vielen sozialen Bereichen (z. B. Krankenhäuser, Pflegedienste) ist die wirtschaftliche Betätigung von Trägern/Institutionen sowie unternehmerisches Denken und Handeln sogar ausdrücklich gewollt. In diesen Bereichen gelten dann unternehmerische Pflichten, z. B. handelsrechtliche Buchführung und Rechnungslegung sowie Steuerpflichten.

Demgegenüber genießen gemeinnützige, nicht wirtschaftliche Vereine $(\mathbb{2} 21 \mathrm{BGB})$ und als gemeinnützig anerkannte Körperschaften $(\mathbb{S} 52$ Abgabenordnung $(\mathrm{AO}))$ die Privilegien, dass sie diesen unternehmerischen Pflichten nicht oder nur eingeschränkt unterliegen, weil sie in ihren hauptsächlichen, die Tätigkeit prägenden Handlungsfeldern nicht wirtschaftlich tätig sein dürfen und gemeinnützige Zwecke verfolgen. Die Begriffe „Gemeinnützigkeit“ und „nicht wirtschaftliche Betätigung“ gehen daher Hand in Hand. In einigen sozialen Handlungsfeldern (z. B. für die Förderung der Träger der freien Jugendhilfe gemäß $\$ 74$ Abs. 1 Nr. 3 SGB VIII) wird ausdrücklich gefordert, dass die Träger gemeinnützige Ziele verfolgen und damit de facto gemeinnützig sein müssen.

\section{Wirtschaftlichkeit versus Gemeinnützigkeit?}

Die „Wirtschaftlichkeit“ hat im allgemeinen Sprachgebrauch und im Kontext unterschiedlicher Rechtsbereiche, etwa im Zivil- oder Gesellschaftsrecht einerseits und im Sozialrecht andererseits, jeweils unterschiedliche Bedeutung.

Bereits bei der Gründung eines Vereins unterscheidet das Bürgerliche Gesetzbuch zwischen dem nicht wirtschaftlichen Verein $(\$ 21 \mathrm{BGB})$ und dem wirtschaftlichen Verein ( $\$ 22$ BGB). Der Zweck des Ersteren ist nicht auf einen wirtschaftlichen Geschäftsbetrieb gerichtet und kann aufgrund einer entsprechenden Satzung durch die Eintragung ins Vereinsregister des zuständigen Amtsgerichtgerichts gegründet werden (e. V.). Der Zweck des wirtschaftlichen Vereins ist auf einen wirtschaftlichen Geschäftsbetrieb gerichtet. Er erlangt seine Rechtsfähigkeit nur durch staatliche Verleihung. Bereits diese Unterscheidung impliziert, dass der Vereinszweck von nicht wirtschaftlichen Vereinen ideeller Natur sein muss. Unter einem wirtschaftlichen Geschäftsbetrieb wird hin- 


\section{Extrablick: Wohlfahrtsverbände in der Legitimationskrise}

gegen die unternehmerische Tätigkeit unter marktwirtschaftlichen Prämissen verstanden, was letztlich auf eine Gewinnerzielungsabsicht zugunsten der Mitglieder hinausläuft. Dies entspricht in der Regel auch dem Zweck einer Gesellschaft mit beschränkter Haftung $(\mathrm{GmbH})$. Diese beliebte Gesellschaftsform für jede Art der wirtschaftlichen Betätigung kann aber, wenn sie ausschließlich und unmittelbar steuerbegünstigte (gemeinnützige) Zwecke verfolgt, auch als gemeinnützig anerkannt werden und die Bezeichnung gGmbH im Firmennamen führen ( $\mathbb{4} 4 \mathrm{GmbHG})$.

In vielen Sozialleistungsbereichen wird dagegen eine „wirtschaftliche Verwendung der Mittel“ (vgl. $\mathbb{S} 74$ Abs. 1 Nr. 2 SGB VIII) oder eine an „den Grundsätzen der Wirtschaftlichkeit und Sparsamkeit orientierte Bemessung der Vergütung “ (vgl. $\$ 179$ Abs. 1 Nr. 3 SGB III, $\mathbb{8} 84$ Abs. 2 SGB XI) gefordert. Dies ist der Tatsache geschuldet, dass in diesen Bereichen die Beitragszahlungen von Versicherten und/oder Steuermittel zur Finanzierung eingesetzt werden. Durch die „wirtschaftliche und sparsame" Verwendung dieser Finanzressourcen sollen die Ausgaben auf das notwendige bzw. angemessene Maß begrenzt werden. Dies wird in der Regel durch entsprechende haushaltsrechtliche Bestimmungen und Maßnahmen sichergestellt (Verwendungsnachweise, Kosten-Leistungs-Rechnungen usw.). Die „Wirtschaftlichkeit“ im unternehmerischen bzw. marktwirtschaftlichen Kontext einerseits und die „Wirtschaftlichkeit“ von Leistungserbringern sozialer Leistungen hat daher unterschiedliche Bedeutung.

Der Begriff „Gemeinnützigkeit“ ist dagegen ein steuerrechtlicher Begriff gem. $\$ 52$ Abs. 1 AO, welcher unabhängig von der Rechtsform der Körperschaft (e. V., $\mathrm{GmbH}$ ) voraussetzt, dass die (Geschäfts-) Tätigkeit darauf gerichtet ist, die Allgemeinheit auf materiellem, geistigem oder sittlichem Gebiet selbstlos zu fördern. Diese Gemeinnützigkeit bestimmt sich in erster Linie nach den in der Satzung bzw. im Gesellschaftsvertrag definierten Unternehmenszwecken. Die Gemeinnützigkeit kann deshalb auch nur bezogen auf Körperschaften verliehen oder entzogen werden, nicht jedoch Einzelpersonen zu- oder aberkannt werden (vgl. $\$ 51$ Abs. 1 $\mathrm{AO}$ ). Aber auch die praktische (Geschäfts-)Tätigkeit ist für die Beurteilung der Gemeinnützigkeit maßgeblich ${ }^{1}$.

\section{Das "Nebenzweckprivileg" als Tor}

zu wirtschaftlicher Betätigung?

Die in $\mathbb{5} 51 \mathrm{AO}$ normierte ,selbstlose Förderung der Allgemeinheit auf materiellem, geistigem oder sittlichem Gebiet“ als Zweck der Tätigkeit erfährt eine kleine Einschränkung über die Definition des Begriffes „Selbstlosigkeit“ ( $\ 55 \mathrm{AO})$. Aus der dortigen Formulierung
„Eine Förderung oder Unterstützung geschieht selbstlos, wenn dadurch nicht in erster Linie eigenwirtschaftliche Zwecke - zum Beispiel gewerbliche Zwecke oder sonstige Erwerbszwecke - verfolgt werden ..." folgt, dass die Tätigkeit nicht ausschließlich selbstlos sein muss. Vielmehr schließt die nachrangige Verfolgung eigenwirtschaftlicher Zwecke ein selbstloses Handeln nicht aus (sog. „Nebenzweckprivileg“). Durch dieses Nebenzweckprivileg führt eine wirtschaftliche Betätigung von untergeordneter Bedeutung nicht automatisch zum Verlust der Gemeinnützigkeit ${ }^{2}$. Die Frage wann und wie lange eine wirtschaftliche Betätigung nur untergeordnete Bedeutung hat, ist einzelfallabhängig. Verallgemeinernd lässt sich sagen, dass von einem Nebenzweck ausgegangen werden kann, wenn der Tätigkeitszweck zu einem deutlich überwiegenden Teil durch nicht unternehmerische Aktivitäten verwirklicht wird und die unternehmerischen Tätigkeiten dem Hauptzweck eindeutig untergeordnet sind (Palandt 2020, 79. Auflage, \$ 21 BGB, RdNr. 7).

Dies hat der $\mathrm{BGH}^{3}$ in einer Entscheidung ausgeführt, die sich auf einen Verein bezieht, der neun Kindertagesstätten mit jeweils 16 bis 32 Kindern betreibt. Der satzungsmäßige Zweck des Vereins mit elf Mitgliedern, ist die „theoretische und praktische Arbeit auf dem Gebiet der Erziehung und Jugendberatung, insbesondere durch Projekte wie die Einrichtung von Elterninitiativ-Kindertagesstätten, durch den Aufbau von beispielsweise Beratungsstellen oder Selbsthilfeprojekten für Jugendliche und junge Erwachsene“. Laut der Entscheidung des BGH stellt der Betrieb der Kindertagesstätten zwar eine unternehmerische Tätigkeit dar, weil der Verein diese Leistung am äußeren Markt planmäßig, dauerhaft und gegen Entgelt anbiete. Diese wirtschaftliche Tätigkeit sei aber dem nichtwirtschaftlichen Hauptzweck des Vereins zu- und untergeordnet und Hilfsmittel zu dessen Erreichung.

\section{Wirtschaftliche Betätigung als Rechtfertigung für hohe (marktwirtschaftliche) Vergütungen?}

Vor dem Hintergrund, dass sich die Anerkennung als gemeinnützige Körperschaft und die wirtschaftliche bzw. unternehmerische Tätigkeit als hauptsächlicher Betätigungszweck ausschließen, erscheint es mehr als zweifelhaft, die wirtschaftliche Betätigung als Rechtfertigung für hohe, der freien Wirtschaft entsprechenden Vergütungen heranzuziehen. Gerade weil durch die Anerkennung als gemeinnützige Körperschaft die Führungskräfte nicht gezwungen sind, Gewinne zur Privatnützigkeit der Gesellschafter zu erwirtschaften und zudem aufgrund der steuerrechtlichen Privilegien (z. B. bei der Körperschafts-, Gewerbe- und Umsatzsteuer) von 
entsprechenden unternehmerischen Pflichten entlastet sind, kann die Verantwortung für gemeinnützige Organisationen nicht ohne weiteres mit der für unternehmerische Tätigkeiten gleichgesetzt werden.

Dies bedeutet jedoch nicht, dass Führungskräfte in gemeinnützigen Körperschaften keine gesteigerte Verantwortung tragen würden. Auch die wirtschaftliche Führung von gemeinnützigen Körperschaften nach dem sozialrechtlichen Verständnis stellt an Führungskräfte hohe Anforderungen. Allerdings sind an die Bemessung der Vergütungen ${ }^{4}$ andere Maßstäbe anzulegen. So darf die gemeinnützige Körperschaft „keine Person durch Ausgaben, die dem Zweck der Körperschaft fremd sind, oder durch unverhältnismäßig hohe Vergütungen begünstigen“ ( $\$ 55 \mathrm{Abs} .1 \mathrm{Nr}$. $3 \mathrm{AO}$ ), weil ansonsten das Merkmal der „Selbstlosigkeit“ entfiele (vgl. \$S 52, 55 AO). Für die Frage, wie eine verhältnismäßige Vergütung von Führungskräften bestimmt werden kann, können etablierte Bemessungsgrundsätze für Vergütungen (z. B. tarifliche Eingruppierungsmerkmale) oder sozialrechtliche Normen (z. B. $\$ 84$ Abs. 2 Satz 5, 6 SGB XI) herangezogen werden:

„Die Bezahlung von Gehältern bis zur Höhe tarifvertraglich vereinbarter Vergütungen sowie entsprechender Vergütungen nach kirchlichen Arbeitsrechtsregelungen kann dabei nicht als unwirtschaftlich abgelehnt werden. Für eine darüber hinausgehende Bezahlung bedarf es eines sachlichen Grundes“ ( $\mathbb{8} 84$ Abs. 2 Satz 5, 6 SGB XI). Als Sachgrund für eine höhere, übertarifliche Bezahlung von Führungskräften könnte beispielsweise das Maß der Verantwortung in der jeweiligen Leitungsfunktion herangezogen werden (z. B. Anzahl und Qualifikation der unmittelbar unterstellten Mitarbeiter_innen, Entscheidungsverantwortung personeller, finanzieller und strategischer Art, Vertretungsbefugnisse), nicht allein jedoch der Umstand, dass im Rahmen des Nebenzweckprivilegs auch unternehmerisches Handeln erforderlich ist.

\section{Legal und beyond compliance}

Über- und außertarifliche Vergütungen sind rechtlich somit nicht ausgeschlossen und stehen dem Merkmal der „Selbstlosigkeit“ aus juristischer Perspektive auch nicht zwingend entgegen, erscheinen jedoch in besonderer Weise legitimierungspflichtig. Die öffentliche Diskussion, die das Bekanntwerden besonderer Privilegien oder hoher Gehälter von Manager_innen von Sozialverbänden erzeugt, zeigt darüber hinaus, dass eine ausschließlich juristische Prüfung zu kurz greift. Geschäftspraktiken einer Organisation können der „legal compliance“ entsprechen, also gesetzeskonform sein (Vilain 2008, S. 138), und die Organisation dennoch belasten, wenn sie keine Akzeptanz in der Gesellschaft, bei Unterstützer_innen sowie bei Geld- und Auftraggeber_innen finden. Der Eindruck einer gesellschaftlichen Entfremdung des Managements entzieht gemeinnützigen Organisationen die Basis ihrer Integrität als zivilgesellschaftliche Akteure, als „Kristallisationskern[e] für freiwilliges und bürgerschaftliches Engagement in seinen vielfältigen Formen und Ausprägungen “ (BAGFW 2018). Sie schaden dann der sozialen und ökonomischen Nachhaltigkeit der Organisation, weil sich das Risiko erhöht, dass das Engagement in der und für die Organisation, die Unterstützung durch Mitgliedschaften und (politische) Fürsprache sowie finanzielle Förderungen und Aufträge zurückgehen.

Insofern ist es aus einer sozioökonomischen Perspektive im eigenen Interesse einer Organisation, die unternehmerischen Prozesse normativ „im Sinne einer beyond compliance" (Vilain 2008, S. 139) zu rahmen, also operative und strategische Geschäftspraktiken mit der verlautbarten Mission der Organisation in Einklang zu bringen. In der Entwicklung eines entsprechenden Leitbildes oder Governance Codex geht es zunächst darum, zu definieren

- in welchen sozialen und ökonomischen Spannungsfeldern sich normative Festlegungen bewegen,

- in welche Wechselbeziehungen und Abhängigkeiten die Organisation eingebunden ist sowie

- in welchen Arenen (Strauss 19935) durch welche Stakeholder die Relevanz von und die Ansprüche an gemeinnützige Organisationen ausgehandelt werden.

\section{Corporate Social Responsibility als Heraus- forderung für soziale Organisationen}

Prägte gemeinnützige Organisationen - insbesondere die anerkannten Verbände der freien Wohlfahrtspflege bislang scheinbar der „Habitus der ,per se Guten“ “ (Vilian 2008, S. 136; vgl. auch Stoll und Hermann 2020, S. 12), sind sie nicht zuletzt durch das öffentliche Aufsehen um Privilegien und Gehälter des Managements, aber auch um „Vetternwirtschaft“, schlechte Arbeitsbedingungen der Mitarbeiter_innenschaft an der Basis oder Pflege- und Missbrauchsskandale gefordert, ihre „Corporate Social Responsibility“ (CSR), also ihren Beitrag zur gesellschaftlichen Weiterentwicklung, unter Beweis zu stellen und entsprechend Compliance-Prozesse anzustoßen. Dies betrifft bei Weitem nicht nur, aber auch die Frage, mit welcher Haltung das Management in Bezug auf die eigene Rolle auftritt.

CSR-Konzepte beziehen sich sowohl auf die Außenwirkung einer Organisation als auch auf interne Prozesse und Wirkungen (Stoll und Hermann 2020, S. 12). Diese beiden Dimensionen lassen sich vermutlich in den meis- 


\section{Extrablick: Wohlfahrtsverbände in der Legitimationskrise}

ten Organisationen kaum voneinander trennen. In sozialen Organisationen sind sie aber u. a. auch deshalb eng verwoben, weil die Beschäftigten nicht selten auch Mitglieder, Ehrenamtliche, politische Fürsprecher_innen und Repräsentant_innen, also Multi-Stakeholder sind, die im Innen und Außen der Organisation wirken. Wenn in CSR-Konzepten davon ausgegangen wird, dass „, $[\mathrm{k}]$ eine Organisation [...] langfristig überleben [...] bzw. im Interesse ihrer diversen Stakeholder handeln [kann], wenn der Fokus entweder z. B. nur auf der eng verstandenen ökonomischen Nachhaltigkeit oder nur auf der eng verstandenen sozialen Dimension liegt "6 (ebd, S. 15), dann bedeutet dies im Kern zunächst zweierlei:

1. Sowohl die Interessen der unterschiedlichen Stakeholder, also Beteiligungsgruppen, müssen in Balance zueinander gebracht als auch die komplexen Rollen mancher Stakeholder berücksichtigt werden.

2. Es geht nicht um eine von Eigeninteressen unabhängige soziale Verantwortung der Organisation, sondern um die nachhaltige Stabilität der Organisation in allen Dimensionen (vgl. Lochner 2020, S. 325).

Freeman, ein zentraler Vertreter von Stakeholder-Ansätzen in der Diskussion um die Nachhaltigkeit von Unternehmen, geht etwa davon aus, dass es vor dem Hintergrund der Ungewissheit gesellschaftlicher Entwicklungen für den langfristigen Bestand von Organisationen unabdingbar ist, Organisationsentscheidungen und -prozesse hinsichtlich ihrer Wirkung auf das gesamte Beziehungsnetzwerk zu reflektieren. Es könne schließlich nicht zuverlässig eingeschätzt werden, wie sich Einflussmöglichkeiten einzelner Stakeholdergruppen verschieben (vgl. Freemann 2010, S. 46; 2011, S. 226). Insofern sei es auch ökonomisch kurzsichtig, die Perspektiven von Gruppen mit gegenwärtig marginalem Einfluss zu ignorieren. Mit Bezug auf die Offenlegung umstrittener Managementprivilegien zeigt sich zum Beispiel, dass die öffentliche Debatte in den Medien z. T. von „Insidern“, z. B. Vorstandsmitgliedern oder ehemaligen Mitarbeiter_innen, befördert wird, deren Einflussmöglichkeiten bis dato möglicherweise unterschätzt wurden.

\section{Sozial-gemeinnützige Organisationen} als gesellschaftliche Akteure

In den bestehenden Arenen, in denen die gesellschaftliche Relevanz von sozial-gemeinnützigen Organisationen und damit verbundene Handlungsprämissen verhandelt werden, geht auch diese Perspektive nicht allen Beteiligten weit genug. Vielmehr herrscht insbesondere bezüglich der in vielen CSR-Konzepten vertretenen These der Vereinbarkeit ökonomischer und sozialer Interessen, die auch den Ansatz Freemans prägt, Uneinigkeit. Kritisiert wird an dieser im Kern liberalen Perspektive, dass soziale Ziele damit immer einem Rationalisierungsdruck unterlägen. Sie würden unabhängig von ihrer gesellschaftlichen Relevanz nur dann handlungsleitend für die Organisation, wenn sich daraus ein marktstrategischer Vorteil ergibt (Lochner 2020, S. 326). Gehälter und Privilegien des Managements müssten also nicht nur (kurzfristig) für Aufsehen und Empörung in der Gesellschaft sorgen, sondern tatsächlich dazu führen, dass der betreffenden Organisation daraus wirtschaftliche Nachteile entstehen.

Dieser marktwirtschaftlichen Logik könne nur durch überorganisationale Absprachen oder Regelungen begegnet werden, die den Rationalisierungsdruck mildern, weil sie verbindliche Standards für alle und damit Raum für „andere Handlungsorientierungen“ schaffen (vgl. Klatetzki und Nokielski 2010, S. 36). Mit Blick auf die Vergütung des Managements ist zwar äußerst fraglich, ob diese über den oben skizzierten rechtlichen Rahmen hinaus von außen reguliert werden kann und soll. Ein gemeinsamer Standard könnte sich jedoch auch auf die Erwartung an unternehmerische Transparenz sozial-gemeinnütziger Organisationen beziehen. Sie würde eine Basis dafür schaffen, dass alle Stakeholder einer Organisation in die Lage versetzt werden, ,ihr Recht auf Konflikt" (Wagner 2013, S. 82) auszuüben, d. h. über die Ausrichtung des Managements zu streiten und Bedingungen der gesellschaftlichen Anerkennungen von Gemeinnützigkeit auszuhandeln. Dies wiederum würde auch die Position sozial-gemeinnütziger Organisationen als zivilgesellschaftliche Akteure stärken.

Eingegangen. 4. Juni 2021

Angenommen. 21. Juli 2021

Funding. Open Access funding enabled and organized by Projekt DEAL.

Open Access. Dieser Artikel wird unter der Creative Commons Namensnennung 4.0 International Lizenz veröffentlicht, welche die Nutzung, Vervielfältigung, Bearbeitung, Verbreitung und Wiedergabe in jeglichem Medium und Format erlaubt, sofern Sie den/die ursprünglichen Autor(en) und die Quelle ordnungsgemäß nennen, einen Link zur Creative Commons Lizenz beifügen und angeben, ob Änderungen vorgenommen wurden.

Die in diesem Artikel enthaltenen Bilder und sonstiges Drittmaterial unterliegen ebenfalls der genannten Creative Commons Lizenz, sofern sich aus der Abbildungslegende nichts anderes ergibt. Sofern das betreffende Material nicht unter der genannten Creative Com- 
mons Lizenz steht und die betreffende Handlung nicht nach gesetzlichen Vorschriften erlaubt ist, ist für die oben aufgeführten Weiterverwendungen des Materials die Einwilligung des jeweiligen Rechteinhabers einzuholen.

Weitere Details zur Lizenz entnehmen Sie bitte der Lizenzinformation auf http://creativecommons.org/licenses/by/4.0/deed.de.

1. So konnte beispielsweise der ADAC im Jahr 2017 nur durch eine umfassende Umstrukturierung und der Auslagerung von wirtschaftlichen Tätigkeitsfeldern seine Gemeinnützigkeit erhalten (Spiegel Online 2017).

2. Z. B. Veranstaltung von Vereinsfesten sowie der Verkauf von Speisen und Getränken, um Einnahmen für den Verein zu generieren.

3. BGH-Beschluss vom 16.05.2017, Az: II ZB 7/16.

4. Zur Vergütung zählen neben dem Entgelt auch alle geldwerten Leistungen (hochwertige Dienstwagen, Vergünstigungen, Prämien, Boni usw.).

5. Arenen werden mit Anselm Strauss als soziale Räume verstanden, die durch offene, disparate Fragen entstehen. Aushandlungsgegenstand sind Grundfragen des organisationalen Handelns, die sowohl von außen an die Organisation herangetragen als auch intern aufgeworfen werden können (Strauss 1993, S. 227).

6. CSR umfasst in der Regel nicht nur Fragen der sozialen und ökonomischen Nachhaltigkeit, sondern bezieht insbesondere auch den Beitrag von Unternehmen zur ökologischen Nachhaltigkeit mit ein. Wenn in den vorliegenden Ausfübrungen dieser Aspekt aufgrund des thematischen Fokus' keine Berücksichtigung findet, bedeutet dies nicht, dass sozial-gemeinnützige Organisationen davon befreit wären, sich Gedanken um ibren ökologischen Fußabdruck zu machen. Dies ist mitnichten ein "Sonderthema“, das separat behandelt werden sollte, da in Konzepten der CSR davon ausgegangen wird, dass sich gesellschaftliche Stabilität nur in der Trias von sozialer, ökologischer und ökonomischer Nachhaltigkeit denken lässt (vgl. Stoll 2020, S. 48). Die Beschränkung auf soziale und ökonomische Aspekte stellt insofern eine Komplexitätsreduktion dar, welche zugunsten der argumentativen Stringenz vorgenommen wurde.

\section{Literatur}

AWO AJS (2020). Persönliche Stellungnahme der Geschäftsführung der AWO AJS gGmbH zum Entwurf des Prüfberichtes des AWO Bundesverbandes. https://www.awo-ajs-thueringen.de/aktuelles/news-detail/news/persoenliche-stellungnahme-der-geschaeftsfuehrung-der-awo-ajs-ggmbh-zumentwurf-des-pruefberichtes-des-a/. Zugegriffen: 20. Mai 2020.

BAGFW (2018). Die Freie Wohlfahrtspflege - innovativ und nachhaltig für sozialen Zusammenhalt. Berlin. https://www.bagfw.de/fileadmin/user_upload/Veroeffentlichungen/Publikationen/bagfw Imageflyer_2018_297x420_digital.pdf. Zugegriffen: 15. Juni 2021.

Bastian, V. (2018). Personalauswabl in der Sozialen Arbeit. Eine empirische Studie zum Suchen und Finden pädagogischer Fachkräfte. Wiesbaden: Springer VS.

Freeman, R. E. (2010). Strategic management. A stakeholder approach. Cambridge: University Press.

Freeman, R. E. (2011). Some thoughts on the development of stakeholder theory. In R. A. Phillips (Hrsg.), Stakeholder theory. Impact and prospects (S. 212-233). Cheltenham: Edward Elgar Pub.

Jüttner, J. (2010). Maserati-Mann empört Obdachlosenhelfer. https:// www.spiegel.de/panorama/kritik-an-400-ps-sportwagen-maserati-mannempoert-obdachlosenhelfer-a-678856.html. Zugegriffen: 10. Apr. 2021.

Klatetzki, T., \& Nokielski, H. (2010). Soziale personenbezogene Dienstleistungsorganisationen als bürokratisch-professionelle Handlungszusammenhänge: Weber und die Folgen. In T. Klatetzki (Hrsg.), Soziale personenbezogene Dienstleistungsorganisationen. Soziologische Perspektiven (S. 25-60). Wiesbaden: Springer VS.
Lochner, B. (2020). Adressat*innen als Stakeholder pädagogischer Organisationen. (unter Mitarbeit von Tom Witton \& M. Kim). In P. Cloos, B. Lochner \& H. Schoneville (Hrsg.), Das Projekt der Sozialen Arbeit (S. 323335). Wiesbaden: Springer VS.

Palandt, \& Ellenberger, J. (2020). Kommentar zum Bürgerlichen Gesetzbuch (79. Aufl.). München: Beck.

Reinhardt, C. (2014). Von der Maserati-Affäre zum Treberhilfe-Skandal. https://www.rbb24.de/politik/hintergrund/Hintergrund-Maserati-AffaereTreberhilfe-Skandal.html. Zugegriffen: 10. Apr. 2021.

Reinhardt, D., \& Reißland, A. (2020). AWO außer Kontrolle - Selbstbedienung in der Thüringer AWO. https://www.mdr.de/nachrichten/thueringen/video-exakt-die-story-awo-ausser-kontrolle-selbstbedienung-in-derthueringer-awo-100_zc-1201c59b_zs-4e0ede05.html. Zugegriffen: 10. Apr. 2021.

Schönball, R. (2011). Finanzamt erkennt Treberhilfe die Gemeinnützigkeit ab. https://www.tagesspiegel.de/berlin/sozialeinrichtungen-finanzamterkennt-treberhilfe-die-gemeinnuetzigkeit-ab/3704128.html. Zugegriffen: 10. Apr. 2021

Spiegel Online (2017). ADAC darf Verein bleiben. https://www.spiegel. de/auto/aktuell/adac-darf-verein-bleiben-a-1131483.html (Erstellt: 24. Jan. 2017). Zugegriffen: 21. Juli 2021.

Stoll, B. (2020). Ansätze zur Verankerung von CSR in profit- und sozialwirtschaftlichen Unternehmen. In B. Stoll \& H. Hermann (Hrsg.), Corporate Social Responsibility - Impulse aus der und für die Profit-und Sozialwirtschaft (S. 47-72). Opladen, Berlin, Toronto: Barbara Budrich.

Stoll, B., \& Hermann, H. (2020). Was Corporate Social Responsibility ist... hängt von ihrer Rahmung ab. In B. Stoll \& H. Hermann (Hrsg.), Corporate Social Responsibility - Impulse aus der und für die Profit-und Sozialwirtschaft (S. 11-20). Opladen: Budrich.

Strauss, A. (1993). Continual permutations of action. New York: De Gruyter.

Vilain, M. (2008). Corporate Social Resonsibility ein Thema für die Sozialwirtschaft? In A. Markert, A. Buckley, M. Vilain \& M. Biebricher (Hrsg.), Soziale Arbeit und Sozialwirtschaft. Beiträge zu einem Feld im Umbruch (S. 135-158). Münster: LIT.

Wagner, T. (2013). Soziale Arbeit, Bürgerschaft und (Post-)Demokratie. Widersprüche, 130, 61-86. 